\title{
Radiology residents' experience with intussusception reduction
}

\author{
Cyrus Bateni • Rebecca Stein-Wexler • \\ Sandra L. Wootton-Gorges $\cdot$ Chin-Shang Li
}

Received: 25 July 2010 /Revised: 17 October 2010 / Accepted: 1 November 2010 /Published online: 22 December 2010

(C) The Author(s) 2010 This article is published with open access at Springerlink.com

\begin{abstract}
Background Residents should be exposed to adequate procedural volume to act independently upon completion of training. Informal inquiry led us to question whether residents encounter enough intussusception reductions to become comfortable with the procedure.

Objective We sought to determine radiology residents' exposure to intussusception reductions, and whether their experiences vary by region or institution.

Materials and methods U.S. radiology residency program directors were asked to encourage their residents to complete a 12-question online survey describing characteristics of their pediatric radiology department, experiences with intussusception reduction, and confidence in their own ability to perform the procedure.

Results Six hundred sixty-four residents responded during the study period. Of those, 308 (46.4\%) had not experienced an intussusception reduction, and 228 (34\%) had experienced only one or two. Twenty-two percent of fourthyear residents had never experienced an intussusception reduction, and $21 \%$ had experienced only one. Among second- through fourth-year residents, only 99 (18.3\%) felt confident that they could competently reduce an intussusception $(P<0.0001)$, and $336(62.2 \%)$ thought they would
\end{abstract}

C. Bateni $(\varangle) \cdot$ R. Stein-Wexler $\cdot$ S. L. Wootton-Gorges

Department of Radiology, University of California at Davis,

4860 Y St., Suite 3100,

Sacramento, CA 95817, USA

e-mail: rebecca.steinwexler@ucdmc.ucdavis.edu

C.-S. Li

Department of Public Health Sciences, Division of Biostatistics, University of California at Davis,

Davis, CA, USA benefit from a computer-assisted training model simulating intussusception reduction $(P<0.0001)$.

Conclusion Radiology residents have limited opportunity to learn intussusception reduction and therefore lack confidence. Most think they would benefit from additional training with a computer-simulation model.

Keywords Intussusception reduction - Resident . Radiology Education

\section{Introduction}

The goal of radiology training is to prepare residents to practice competently and independently. During residency, they are usually exposed to a sufficient volume and variety of cases so that educators can certify them competent to practice radiology after 4 years. However, analysis of our institution's volume of intussusception cases, along with an informal survey of our and nearby residents' exposure to intussusception, led us to question whether we are adequately training radiology residents to perform intussusception reduction, and whether our experience reflects a nationwide problem or is unique to our geographic area or type of practice. Are residents encountering enough intussusception to become confident in their ability to perform reductions?

Intussusception is a significant cause of acute abdomen in young children, with between 0.5 and 2.3 cases per 1,000 live births. Radiologists who succeed at reducing intussusceptions provide an essential service $[1,2]$. Prior exposure to this procedure improves a radiologist's likelihood of success [3]. Although some children are treated by trained pediatric radiologists with extensive experience in reduction, $39 \%$ of pediatric patients present to community 
hospitals and may be treated by radiologists who haven't had fellowship training in the specialty [3].

We developed a survey to evaluate resident exposure to intussusception reduction nationwide, correlating the level of training with the number of intussusception reductions. We also determined the characteristics of different training situations to establish whether specific institution types are more or less successful at exposing residents to intussusceptions. Finally, we asked whether residents felt competent to reduce an intussusception, and whether they think they would benefit from computer-simulated training.

\section{Materials and methods}

This study was reviewed and approved by our institution's IRB. A 12-question survey was created utilizing www.surveymonkey.com (Fig. 1). Most questions allowed for only one best answer, and most did not demand an answer in order for the participant to submit the survey. However, a single question was mandatory (Question \#9: How many intussusception reductions have you been involved with?). Question \#10 ("These reductions have employed...") could have more than one response, reflecting the variety of approaches to intussusception reduction. These include the use of fluoroscopy or US to monitor the reduction and either liquid or air as the agent to reduce the intussusception.

Radiology residency program directors throughout the United States were contacted through the Association of
Program Directors in Radiology via e-mail in August 2009 and asked to send their residents a link to the survey. A reminder was sent about 2 weeks later. We analyzed data gathered over a 4-week time span, excluding responses submitted after the deadline.

\section{Results}

A total of 684 residents completed the survey. Twenty were excluded as they responded after the period of data collection. The 664 respondents were from programs in 32 states and the District of Columbia, representing 14.9\% of 4,455 residents nationwide [4]. One hundred twentythree $(18.5 \%)$ were in their first year of radiology training (R1), $168(25.3 \%)$ in their second year (R2), 178 (26.8\%) in their third year (R3), and 195 (29.4\%) in their fourth year (R4). Programs in all regions of the United States were represented.

The respondents learn pediatric radiology predominantly at children's hospitals that are either freestanding (29\%) or embedded within a university hospital (54\%), with the other $17 \%$ at community hospitals and other institutions.

With the exception of small programs, there was a fairly even distribution of residents with respect to radiology residency size. One hundred fifty-two respondents were in programs with 11-20 residents, 159 with $21-30$ residents, 168 with $31-40$ residents, and 155 in programs with greater than 40 residents. Only 30 were in programs with fewer than 11 residents.
Fig. 1 Questionnaire submitted to U.S. radiology residents
Questionnaire

1. What year of radiology residency training are you in?

2. What state is your residency located in?

3. How many radiology residents are in your program?

4. How would you characterize the facility where you learn pediatric radiology?

5. How many staff pediatric radiologists work at this institution?

6. How many radiology residents usually rotate on pediatric radiology at a time?

7. How many weeks of formal pediatric radiology training have you had thus far during your radiology residency?

8. How many cases of intussusception have you seen on CT or Ultrasound during residency?

9. How many intussusception reductions have you been involved with?

10. These reductions have employed:

11. Are you confident that you can competently reduce an intussusception by yourself?

12. Do you think you would benefit from a computer-assisted training model that would simulate employing air to reduce an intussusception? 
Exposure to intussusception vs. residency year and weeks of pediatric radiology training

We found a statistically significant correlation between the number of intussusceptions experienced and the year of residency ( $P$-value $<0.0001$, Jonckheere-Terpstra test), as well as a statistically significant correlation between the number of weeks of formal pediatric radiology training and intussusception exposure $(P$-value $<0.0001$, Jonckheere-Terpstra test). Of 195 fourth-year radiology residents, $22 \%$ had no experience with intussusception reduction, and $21 \%$ had seen only one case (Table 1 ). On average, fourth-year residents had seen roughly two intussusception reduction cases. (Some responses included a range; therefore, average intussusception exposures are approximations.)

As residents advance through training, the number of weeks of pediatric radiology training increases. The vast majority of first-year residents had minimal radiology training, and their responses were excluded from much of our analysis. Of third-year residents, $21 \%$ had 0 4 weeks of training, 40\% had 5-8 weeks, and 31\% had 9-12 weeks. Nearly half (43\%) of fourth-year residents had 9-12 weeks of pediatric radiology, whereas $26 \%$ had more than 12 weeks; $21 \%$ had only $5-8$ weeks. Table 2 analyzes residents' experience with intussusception reduction based on the number of weeks they had rotated on pediatric radiology. As would be expected, experience with intussusception reduction increases with weeks of pediatric radiology. Those with $0-4$ weeks of pediatric radiology averaged roughly 0.5 reductions, whereas those with more than 12 weeks of training averaged about 2.5 reductions.

The survey also addressed residents' opportunity to diagnose intussusception on cross-sectional imaging studies. Of the 541 second- through fourth-year radiology residents (11 residents did not respond), the majority (361, or $66.7 \%$ ) had seen fewer than five cases on CT, US. One hundred thirty-five $(25.0 \%)$ had seen 5-10 cases, and 34 $(6.3 \%)$ had seen more than 10 . Fourth-year radiology residents were more experienced than their counterparts with less training. However, the majority of seniors (102, or $52.3 \%$ ) had diagnosed fewer than five cases of intussusception on CT or US, whereas 68 (34.9\%) had seen 5-10 cases.

\section{Regional variations}

Residents were divided into four regions of the United States (Midwest, Northeast, South and West) based upon the United States Census Bureau protocol [5]. There were 158 respondents from the Midwest, 228 from the Northeast, 165 from the South, and 102 from the West (11 residents chose not to respond). Residents in these different regions were exposed to a similar number of intussusception reductions ( $P$-value 0.8237 , Kruskal-Wallis test).

Analysis of regional differences in intussusception reduction technique was also performed (air, liquid, fluoroscopy, US). As multiple responses were allowed, the number of answers (719) exceeded the number of respondents (664); also, some chose not to answer this question. Two hundred seventy-six (41.6\%) intussusception reductions used fluoroscopy with air, 135 (20.3\%) used fluoroscopy with liquid, 27 (4.2\%) used fluoroscopy with air and liquid, and $10(1.4 \%)$ used US with liquid (10 replied "unsure" and 261 replied "not applicable"). Interestingly, intussusception reduction technique appears to vary regionally. Fluoroscopy and air is much more likely than liquid to be employed in the Northeast and South (43.0\% and 46.8\%, respectively) and slightly more commonly in the Midwest (32.8\%), but fluoroscopy and liquid is more common in the West $(31.8 \%)(P$-value $<0.0001$, chi-square test $)$.

Potential differences based upon institution

Institution characteristics were evaluated to establish whether a resident's exposure to intussusception is related to the size or type of institution where they learn pediatric radiology. One hundred seventy-five train at a freestanding children's hospital, 343 at a children's hospital within a university hospital, 71 at a community hospital, and 47 at "other." Twenty-eight chose not to answer this question.
Table 1 Radiology residents' experience with intussusception reduction during residency training

\begin{tabular}{lcrcc}
\hline Number of reductions & & & \\
\hline Radiology residency training level & 0 & 1 & 2 & 3 or more \\
\hline R1 & $110(89.4 \%)$ & $12(9.8 \%)$ & $1(0.8 \%)$ & $0(0 \%)$ \\
R2 & $99(58.9 \%)$ & $38(22.6 \%)$ & $18(10.7 \%)$ & $13(7.7 \%)$ \\
R3 & $56(31.5 \%)$ & $41(23.0 \%)$ & $33(18.5 \%)$ & $48(27.0 \%)$ \\
R4 & $43(22.1 \%)$ & $41(21.0 \%)$ & $44(22.6 \%)$ & $67(34.4 \%)$ \\
Total & 308 & 132 & 96 & 128 \\
\hline
\end{tabular}


Table 2 Residents' experience with intussusception reduction based on weeks of radiology rotation

\begin{tabular}{lcccc}
\hline Number of reductions & & & \\
\hline Weeks of formal pediatric radiology & 0 & 1 & 2 & 3 or more \\
\hline $0-4$ & $192(74.4 \%)$ & $35(13.6 \%)$ & $19(7.4)$ & $12(4.7 \%)$ \\
$5-8$ & $71(38.8 \%)$ & $52(28.4 \%)$ & $26(14.2 \%)$ & $34(18.6 \%)$ \\
$9-12$ & $34(21.8 \%)$ & $34(21.8 \%)$ & $35(22.4 \%)$ & $53(34.0 \%)$ \\
$>12$ & $10(15.4 \%)$ & $11(16.9 \%)$ & $16(24.6 \%)$ & $28(43.1 \%)$
\end{tabular}

We found no significant difference among groups with regard to the facility type and the number of intussusception reductions ( $P$-value 0.33 , Kruskal-Wallis test). Specifically, analysis of fourth-year radiology residents showed that of the 65 of those radiology residents training at a pediatric hospital, 26/65 (40\%) had experienced 0-1 reductions, and $13(20 \%)$ had experienced 2 reductions. Of the 103 fourthyear residents training at a pediatric facility embedded within a university hospital, 41/103 (39.8\%) had experienced 0-1 reductions, and $29(28.2 \%)$ had participated in 2. Of those 20 who train at a community hospital, $11(55 \%)$ have seen $0-1$ reduction, and $2(10 \%)$ have experienced 2 reductions.

The primary method employed for reduction in freestanding children's hospitals and children's hospitals embedded within a university hospital was fluoroscopy with air (which was employed about twice as often as liquid). However, residents training at community hospitals were more likely to be exposed to liquid reduction than to air reduction ( $P$-value 0.0058 , chi-square test).

Most residents train with 10 or fewer pediatric radiologists: 163 residents were at institutions with 1-2 pediatric radiologists, 227 with 3-5 pediatric radiologists and 136 with 6-10. Forty-two trained with 11-20 pediatric radiologists and 37 with more than 20 (59 were unsure). Exposure to intussusception reduction did not vary at these different institutions.

When program size was assessed by determining the number of residents rotating on pediatric radiology at a time, there was also no statistically significant difference between the number of residents and their exposure to intussusception reduction ( $P$-value 0.1085 , Jonckheere-Terpstra test). Programs with two residents at a time were most common $(42 \%)$; others usually had either one (21\%) or three $(19 \%)$.
Resident confidence and interest in simulation training

The majority of junior and senior residents are not confident in their ability to reduce intussusception; this correlates with the number of intussusception reductions they have performed. Of the 541 second- through fourthyear residents who answered Question \#11 ("Are you confident that you can competently reduce an intussusceptions by yourself?"), only 99 (18.3\%) responded yes. Three hundred eleven (57.5\%) replied no, and 131 (24.2\%) answered maybe $(P$-value $<0.0001$, chi-square test). The analysis of fourth-year residents only shows that they, too, lack confidence in their ability to reduce intussusception. Only $52(26.7 \%)$ were confident that they could reduce an intussusception, whereas $89(45.6 \%)$ were not, with the remaining 54 (27.7\%) unsure.

A contingency table was used to compare the number of intussusception reductions with resident confidence, and there is a statistically significant correlation between these groups ( $P$-value $<0.0001$, Wilcoxon rank sum test). Residents who were confident in their ability to reduce an intussusception have had a statistically significantly higher number of intussusception reductions during training than those who were not confident or were unsure (Table 3).

Residents at all training levels are interested in a computer model simulating the use of fluoroscopy and air to reduce an intussusception. In the second- through fourthyear cohort, $336(62.2 \%)$ responded positively to this question $(P$-value $<0.0001$, chi-square test), 88 (16.3\%) replied "no," and 116 (21.5\% were unsure). One person did not answer this question. Answers were similar when fourth-year radiology residents were isolated: 120 (61.5\%) were interested in a computer model, $35(17.9 \%)$ were not, and $40(20.5 \%)$ were unsure. Overall, resident interest in a
Table 3 Number of intussusception reductions compared with resident confidence level

\begin{tabular}{lccccc}
\hline \multicolumn{5}{l}{ Number of intussusception reductions } \\
\hline Confidence & 0 & 1 & 2 & 3 or greater & Total \\
\hline Yes & $2(0.3 \%)$ & $14(2.1 \%)$ & $19(2.9 \%)$ & $69(10.4 \%)$ & $104(15.7 \%)$ \\
No & $272(41.1 \%)$ & $88(13.3 \%)$ & $38(5.7 \%)$ & $16(2.4 \%)$ & $414(62.6 \%)$ \\
\hline
\end{tabular}


computer model was inversely correlated with their exposure to intussusception ( $P$-value 0.0077 , Wilcoxon rank sum test).

\section{Discussion}

Diagnosis and treatment of intussusception is an important part of residency training. This nationwide survey shows there are wide variations in resident exposure to intussusception reduction, but that these variations correlate with neither geographic region nor with the size of the training institution. Thus, exposure cannot be increased simply by restricting training to, for example, larger institutions with many pediatric radiologists.

Not surprisingly, resident exposure to intussusception reduction increases with advancement through residency, correlating positively with both resident year and weeks spent in pediatric radiology. Many first-year residents have had very little pediatric radiology and, correspondingly, few experiences with intussusception reduction, whereas the typical fourth-year resident has experienced more. However, although the typical resident with 9 or more weeks of pediatric radiology has experienced just over two intussusception reductions, exposure is still limited for many ( $20 \%$ had seen none and another $20 \%$ had seen only one).

An unexpected finding is the regional and institutional variation in technique employed for intussusception reduction. Residents in the southern and northeastern United States are most likely to be exposed to air reduction, whereas exposure to air is relatively uncommon in the West (the Midwest is intermediate). Residents training in pediatric radiology at freestanding pediatric hospitals or those within a university hospital are more often exposed to air reduction, while residents at community hospitals tend to experience liquid reduction.

There are several limitations to this study. Although responses were fairly evenly divided among the years of residency, only $14.9 \%$ of 4,455 residents answered the survey. This may result in bias. We had to rely on program directors to circulate the survey and residents to take the time to respond. Also, the study was circulated early in the academic year and, thus, may underestimate resident exposure to intussusception. Some of the responses include a range of exposure to intussusception, limiting the precision of some of our results. The study did not attempt to differentiate between resident observation of and active participation in reduction, and it is quite likely that the number of residents who have actually played an active role in reduction is much smaller. It is also possible that respondents' recall represents their experiences inaccurately.

There may have been some unintended bias in this study. For instance, we postulated that residents would have little experience and comfort with intussusception reduction. At the time of the survey, we were developing an intussusception computer-simulation model, which led to our inquiry about such a device. We did not ask about other nonexperiential learning methods that may help residents perform intussusception reduction.

Limitations aside, radiology residents often (and appropriately) lack confidence in their ability to reduce intussusception independently. Only $18.3 \%$ of second- through fourth-year residents say they feel confident they can reduce an intussusception on their own; this confidence correlates with increasing exposure to reduction.

Given that the typical radiology resident's exposure to intussusception reduction is limited, most are interested in enhanced learning via an intussusception simulation model [6]. Computer simulation is indeed playing an increasing role in training both residents and practicing radiologists in uncommon, new or technically demanding procedures [7-9]. It appears that radiology residents would benefit from the opportunity to practice intussusception reduction in a simulated environment. Other teaching methods, such as lectures, videos, discussion, "how-I-do-it" papers and increased effort to expose residents to real cases may also help address the need for additional education in this technique.

In raising the question of whether residents are adequately prepared to perform intussusception reduction, we are, in essence, asking whether upon graduation they are able to perform this procedure "competently and without direct supervision" (to quote the American College of Graduate Medical Education) [10], or whether all reductions must be performed by pediatric radiologists. What is adequate training? Is it equivalent to competency? Educators wrestle with the question of what makes a person competent to perform a specific procedure, and-more fundamentally - whether satisfactory completion of residency and certification exams establishes that a physician is indeed competent to practice $[11,12]$. Is it enough to "see one, do one, teach one"? Must one perform a prescribed number of procedures? Must a resident complete a checklist to be deemed competent [13]? Must procedural skills be assessed by direct observation [14]? How adequate is selfassessment of competency? To what extent can a simulation model substitute for the real thing? These critical questions are beyond the scope of this paper, but merit further research and discussion.

The definition of competency has recently been refined by the ACGME to include six core competencies for all residency curricula: patient care, medical knowledge, practice-based learning and improvement, interpersonal and communication skills, professionalism and systems-based practice $[11,15]$. The question of competency to perform intussusception reduction clearly falls under the dual competencies of patient care and medical knowledge. 
However, practice-based learning - in which the resident refines his or her ability to perform as a result of educational experiences-and professionalism, which includes the resident's assessment of his or her own abilities, are also critical. Professionalism is at the core of lifelong learning, for continuing medical education depends on honest assessment of one's deficiencies, and then willingness to invest time and resources addressing them. We have given residents the opportunity to assess their training in intussusception reduction, and they report that it is insufficient.

\section{Conclusion}

We found that residents have limited exposure to intussusception reduction, but that this exposure does not vary with institution type or size. Their confidence in performing this procedure independently is appropriately low. Many residents are interested in a computer-simulation model for intussusception reduction to enhance their clinical training.

\begin{abstract}
Acknowledgements Statistical support for this publication was made possible by Grant Number UL1 RR024146 from the National Center for Research Resources, a component of the National Institutes of Health, and NIH Roadmap for Medical Research. Its contents are solely the responsibility of the authors and do not necessarily represent the official view of NCRR or NIH. Information on Re-engineering the Clinical Research Enterprise can be obtained from http://nihroadmap.nih.gov/clinicalresearch/overviewtranslational.asp.
\end{abstract}

Open Access This article is distributed under the terms of the Creative Commons Attribution Noncommercial License which permits any noncommercial use, distribution, and reproduction in any medium, provided the original author(s) and source are credited.

\section{References}

1. Bruce J, Huh YS, Cooney DR et al (1987) Intussusception: evolution of current management. J Pediatr Gastroenterol Nutr 6:663-674

2. Rennels MB, Parashar UD, Holman RC et al (1998) Lack of an apparent association between intussusception and wild or vaccine rotavirus infection. Pediatr Infect Dis J 17:924-925

3. Jen HC, Shew SB (2009) The impact of hospital type and experience on the operative utilization in pediatric intussusception: a nationwide study. J Pediatr Surg 44:241-246

4. FREIDA online specialty training search-Radiology-Diagnostic. Available via Ama-assn.org. https://freida.ama-assn.org/Freida/ user/specStatisticsSearch.do? method=viewDetail\&spcCd=420\& pageNumber $=2$. Accessed 9 April 2010

5. Census Regions and Divisions of the United States. Available via Census.gov. http://www.census.gov/geo/www/us_regdiv.pdf. Accessed 13 March 2010

6. Stein-Wexler R, Sanchez T, Roper GE et al (2010) An interactive teaching device simulating intussusception reduction. Pediatr Radiol 40:1810-1815

7. Seymour NE, Gallagher AG, Roman SA et al (2002) Virtual reality training improves operating room performance: results of a randomized, double-blinded study. Ann Surg 236(458-63):463-464

8. Sedlack RE, Kolars JC, Alexander JA (2004) Computer simulation enhances patient comfort during endoscopy. Clin Gastroenterol Hepatol 2:348-352

9. Satava RM (2009) The revolution in medical education - the role of simulation. J Grad Med Ed 1:172-175

10. ACGME Program Requirements for Graduate Medical Education in Diagnostic Radiology. Available via acgme.org. http://www.acgme. org/acWebsite/downloads/RRC_progReq/420_diagnostic_radiol ogy_07012010.pdf. Accessed 20 Sept 2010

11. Wood BP (2009) Competency-based training: accreditation as a pathway to wisdom. Radiology 252:322-323

12. Epstein RM, Hundert EM (2002) Defining and assessing professional competence. JAMA 287:226-235

13. Chertoff $\mathrm{J}$ (2008) Competency assessment in resident education. Acad Radiol 15:1215-1216

14. Bari V (2010) Direct observation of procedural skills in radiology. AJR 195:W14-18

15. ACGME outcome project. Available via Acgme.org.http://www. acgme.org/outcome/comp/GeneralCompetenciesStandards21307. pdf. Accessed 12 Sept 2010 